\title{
PRÁCTICAS PROFESIONALES Y LA FORMACIÓN EN COMPETENCIAS PARA LA EMPLEABILIDAD
}

\author{
Marta Ruiz-Corbella \\ Maria José Bautista-Cerro Ruiz \\ Miriam García-Blanco \\ Universidad Nacional de Educación a Distancia
}

\begin{abstract}
RESUMEN: En el diseño de los títulos universitarios uno de los puntos fundamentales que avalan su viabilidad es la empleabilidad de sus egresados. Garantizar la inserción en el mercado de trabajo es un objetivo clave promovido desde diferentes organismos, lo que conlleva que en las instituciones universitarias se impulsen iniciativas dirigidas a su logro. Ahora, esta empleabilidad no se logrará sin el aprendizaje de las competencias específicas y genéricas de cada título de Grado que avalen el diseño de su propio proyecto profesional, lo que facilitará aprender a afrontar su paso a la vida laboral con garantía de éxito. Con este objetivo la asignatura Prácticas Profesionales $V$ del Grado en Educación Social de la UNED desarrolla una experiencia de innovación docente dirigida a saber elaborar un Proyecto Profesional propio. La indagación y recopilación de información, el análisis de la misma, la toma de decisiones fundamentadas y la propuesta de este proyecto aportan los elementos clave para gestionar su empleabilidad.

Los resultados de esta experiencia se recogen con una metodología longitudinal "ex post-facto" a través de las calificaciones recibidas y los cuestionarios de satisfacción de los estudiantes. La experiencia a lo largo de 5 cursos académicos avala la formación de nuestros estudiantes en competencias para el empleo, a la vez que su alto grado de satisfacción ante esta formación.
\end{abstract}

PALABRAS CLAVE: Prácticas Profesionales, Proyecto Profesional, inserción laboral, empleabilidad, educación superior.

\section{PROFESSIONAL PRACTICES AND EMPLOYABILITY SKILLS TRAINING}

ABSTRACT: In the design of university degrees one of the fundamental points that guarantees their viability is the employability of its graduates. Guaranteeing insertion in the labor market is one of the key objectives 
promoted by different organizations, which means that, from different university instances, initiatives aimed at their achievement are promoted. Now, this employability will not be attained without learning the specific and generic skills from each Bachelor degree in order to design ones professional project as well as the means to face the transition into the working life with a guarantee of success. With this aim, the subject Professional Practices $V$ of the Degree in Social Education of the UNED develops an experience of teaching innovation directed to know how to elaborate an own Professional Project. The search and collection of information, its analysis, as well as taking well-founded decisions and proposing a professional strategy, provide the key elements to manage their employability for the incorporation into the labor market.

The results of this experience are collected with a longitudinal methodology "ex post-facto" through the score received in this subject and the results of the questionnaires of student satisfaction. The experience throughout 5 academic years supports the training of our students in competencies for employment, as well as their high level of satisfaction with such training.

KEYWORDS: Professional practices, professional strategy, labor market, employability, higher education.

Recibido: 20/02/2018

Aceptado: 17/07/2018

Correspondencia: Marta Ruiz-Corbella, Universidad Nacional de Educación a Distancia, C/ de Juan del Rosal, 14, 28040 Madrid. Email: mruiz@edu.uned.es.

\section{INTRODUCCIÓN}

La preocupación por la inserción profesional de los estudiantes universitarios ha sido una constante en el diseño de los títulos en cualquiera de sus niveles de Grado, Master y Doctorado (González Lorente y Martínez Clares, 2016). Las indicaciones, orientaciones e informes elaborados por la Agencia Nacional de Evaluación de la Calidad y Acreditación (ANECA, 2009), los observatorios de empleabilidad de los egresados universitarios (Michavila, Martínez, Martín-González, García-Peñalvo, y Cruz-Benito, 2016), o la actividad que desarrollan los Centros de Orientación e Información para el Empleo (COIE) de cada universidad (Suárez Lantarón, 2013) son ejemplos de su preocupación por la empleabilidad (Caballero Fernández, LópezMiguens, y Lampón, 2014). Prueba de este interés es cómo, a lo largo de esta última década, la investigación sobre la empleabilidad de los egresados universitarios también ha vivido una notable atención en nuestro país, traducida en un aumento de la producción científica sobre este tema (Álvarez Pérez y López Aguilar, 2012; Suárez Lantarón, 2014; Pineda i Herrero, Ciraso Calí y Armijos Yambay, 2018; Torres-Valdés, Santa Soriano y Lorenzo Álvarez, 2018; Dapía Conde, y Fernández González, 2016; 
Guàrdia-Olmos, Peró-Cebollero, Martínez-Ricart, Cañete-Masse, Turbany-Oset, y Berlanga-Silvente, 2018).

Si atendemos a las memorias de verificación de los títulos de Grado, observamos que se presta especial atención al carácter profesionalizante de cada propuesta, es decir, a su capacidad para formar profesionales que respondan a las demandas de un mercado cada vez más cambiante. Se requiere y evalúa que toda oferta educativa oficial propuesta por una institución de educación superior garantice "que las personas que obtengan una titulación sepan y puedan encajar sus conocimientos en el marco de las necesidades sociales de su entorno, que tengan capacidades que son necesarias para el empleo en la comunidad en la que tendrán que ejercer sus profesiones, (...)" (Beneittone, González y Wagenaar, 2014, p. 18). Es decir, a la hora de formar en competencias debemos tener en cuenta la finalidad de las mismas, que, sin descuidar la vertiente humana y ciudadana, sea marcadamente profesional, lo que exige saber adaptarse a las demandas de un mercado de trabajo y a una sociedad cada vez más inestables y complejos (Torres-Valdés et al., 2018).

Esto supone un nuevo enfoque de los objetivos que debe atender la formación que se imparte en estas instituciones. No es que sea algo totalmente novedoso, ya que estas siempre se han dirigido a la formación de los profesionales que exige la sociedad (médicos, juristas, arquitectos, ingenieros, profesores, etc.). Pero "el aumento del número de titulados universitarios en los últimos 30 años y el aumento de las tasas de desempleo de los titulados en relación a otros niveles educativos han cambiado la situación de privilegio de los universitarios en el mercado laboral, (...)" (Pineda-Herrero, Agud-Morell, y Ciraso-Calí, 2016, p. 145). Tal como afirma el reciente estudio La universidad española en cifras 2015/2016, la tasa de empleo de la población con estudios superiores es del $78,2 \%$, frente al $82,4 \%$ de la OCDE, presentando como "causa nuclear del menor nivel de empleabilidad en la debilidad estructural del mercado de trabajo en España, que en 2015 seguía presentando el segundo nivel de desempleo más alto de las economías europeas, (...)" (Hernández Armenteros y Pérez García, 2017 , p. 89). Sin embargo, tener estudios superiores sigue garantizando un mayor índice de empleabilidad.

Esta situación presenta entornos tremendamente competitivos que todo profesional debe conocer para mejorar su empleabilidad. Ser conscientes de la situación del mercado al que se desea acceder (principales empleadores, requisitos mínimos solicitados, tipos de contratos, nichos emergentes de mercado, posibilidades de emprendimiento, etc.) es un punto de partida que muchos estudiantes obvian a la hora de planificar su futuro profesional (Guàrdia-Olmos et al., 2018).

En definitiva, se requiere formación no solo en las competencias específicas como futuro profesional en un determinado sector, sino también en aquellas que le facilitan el acceso a ese ámbito laboral. Para ello resulta necesario tanto el conocimiento en profundidad de los distintos modos de inserción laboral, como la capacidad de diseñar un proyecto profesional propio identificando las estrategias de desarrollo profesional más adecuadas para su logro (Suárez Lantarón, 2014). 
Ante este nuevo planteamiento en los diseños de las titulaciones, tal como ya indicamos, las instituciones están llevando a cabo diferentes acciones e iniciativas dirigidas a favorecer la inserción laboral de sus egresados bien a través de los COIE, o de los Observatorios para la empleabilidad. Sin embargo, a pesar de los resultados positivos que obtienen estas acciones, no podemos pasar por alto un grave problema detectado: la escasa relación entre estas acciones y la formación que se imparte en las titulaciones, además de su escasa visibilidad y difusión entre profesorado y alumnado (Ruiz Corbellá et al., 2014; Torres-Valdés et al., 2018).

Teniendo en cuenta esta realidad, parece fundamental que se contemplen en el diseño de los títulos, las competencias y los resultados de aprendizaje que faciliten a los estudiantes el diseño de un proyecto profesional eficaz. Entendemos que no se trata de una formación complementaria, sino que debe formar parte de los contenidos específicos del título. A raíz de esta propuesta este artículo presenta la experiencia que a lo largo de los últimos 5 años ha desarrollado la asignatura de Prácticas Profesionales V del Grado en Educación Social de la UNED, centrada en el diseño del proyecto profesional de sus estudiantes. Con esta perspectiva analizamos, en primer lugar, cómo se prepara a nuestros estudiantes universitarios para la empleabilidad y, en especial, en la materia de Prácticas Profesionales como espacio idóneo para formar en esta competencia. En segundo lugar, exponemos unos primeros resultados de la experiencia que hemos desarrollado en esta asignatura. Además, entendemos que si vinculamos la aprobación de los títulos universitarios a la capacidad de inserción profesional de sus egresados, y si estamos introduciendo medidas encaminadas a promover el espíritu emprendedor del universitario (FCYD, 2011), promover este tipo de experiencias docentes en diferentes asignaturas del Grado contribuirán a una mejor inserción laboral de nuestros egresados en el contexto actual (Caballero Fernández et al., 2014; Torres-Valdés et al., 2018).

\section{La preparación para la inserción laboral como responsabilidad de la universidad}

La inserción laboral es un tema complejo que depende de múltiples factores, unos externos al propio sujeto (sociales, económicos, demográficos, etc.), y otros internos en los que confluyen tanto las capacidades de cada individuo como su formación, sus intereses, posibilidades personales, etc. (Pineda i Herrero et al., 2018). Tanto en un caso como en otro es necesario que cada persona sepa identificarlos, recoger la información necesaria, ser capaz de analizarla para poder tomar decisiones, desarroIlar habilidades y actitudes necesarias que todo estudiante requiere para lograr esa inserción en el mercado laboral (McQuaid y Lindsay, 2005). Ante esta compleja realidad, la búsqueda de un empleo no puede dejarse al azar, sino que debemos facilitar a los estudiantes las competencias, herramientas y recursos necesarios para planificar esta acción, siempre ligada a la situación y demandas del contexto en el que vive, de tal modo que sea capaz de afrontar exigencias inmediatas o futuras del mercado de trabajo (Enríquez y Rentería, 2007). Es decir,

los profesionales necesitan los conocimientos y competencias clave impartidos en el Grado para acceder a un puesto de trabajo con un determinado perfil profesional, que 
les facilita una transición suave al mundo laboral. Es decir, disponer de las destrezas necesarias para su búsqueda y selección, logrando un empleo adecuado a la formación recibida y en estrecha relación con la titulación (Ruiz Corbellá et al., 2014, p. 28).

Para ello resulta esencial una formación práctica orientada a desarrollar competencias para la empleabilidad: búsqueda activa de empleo; formación para el autoempleo; desarrollo de competencias específicas para la inserción profesional; la gestión y técnicas de marketing personal; la planificación del propio currículo académico y profesional; la formación en las demandas emergentes del entorno; la promoción de actitudes proactivas; etc. (Ruiz Corbellá y Quintanal, 2012; Dapía Conde y Fernández González, 2016).

\section{La titulación de grado y su relación con la empleabilidad}

Si acudimos al Marco Español de Cualificaciones de la Educación Superior (MECES), comprobamos que el nivel de Grado contiene un claro objetivo profesional. En concreto, señala que sus resultados de aprendizaje deben estar dirigidos, entre otros, a la aplicación de conocimientos en ámbitos profesionales complejos y/o especializados que requieren el uso de ideas creativas e innovadoras. Es decir, ser capaces de desenvolverse en el ámbito profesional propio de su titulación (MECU, 2014). De esta forma, se evidencia que la inserción laboral de los egresados de este nivel debe estar presente, de una u otra forma, en el diseño y gestión de todas estas titulaciones (Caballero Fernández et al., 2014).

A la vez, resulta habitual que la materia de Prácticas Externas del Grado sea valorada como un aprendizaje que facilita la conexión con el mundo laboral (ANECA 2009; Moreno Gené, 2012). Sin embargo, el último informe de empleo de los universitarios (Michavila et al., 2016) confirma la escasa aplicación de esta materia de Prácticas en la formación de competencias para la empleabilidad de sus estudiantes. Aunque debería ser considerada como un espacio fundamental para contrastar los conocimientos, conocer la realidad laboral, adquirir experiencia, a la vez que la mejor conexión entre ambos entornos: el académico y el profesional. Asignatura que, en definitiva, está favoreciendo la coordinación entre oferta formativa y las necesidades del trabajo, incrementando, así, el componente práctico de la titulación (Moreno Gené, 2012; McKinsey \& Co., 2013).

Ahora bien, al revisar el título del Grado en Educación Social de la UNED, objeto de este estudio y, en concreto, los créditos asignados a las Prácticas Profesionales, se comprueba que entre las competencias y los contenidos que imparte no se atiende la formación para la empleabilidad de nuestros estudiantes. Estos valoran muy positivamente la experiencia que adquieren en las Prácticas, ya que conocen y se implican activamente en un contexto real de intervención socioeducativa.

La cuestión es que Ilegan a conocer uno o varios ámbitos en los que podrían desarrollar su vida profesional, pero carecen de los conocimientos y destrezas necesarios para planificar, elaborar y desarrollar un proyecto profesional, que, además tenga en cuenta las repercusiones en su vida personal. Pero no se les forma en los conocimientos y destrezas necesarios para planificar, elaborar y 
desarrollar un proyecto profesional personal, que garantice el lograr ser actor y autor de su propia trayectoria profesional: ser capaz de construir, destruir y reconstruir a lo largo de la vida su proyecto profesional en un constante ejercicio caracterizado por:

- La planificación de ese proyecto, que implica la prospección y reinterpretación, la reflexión sobre lo pasado y los datos recogidos en su contexto para la toma de decisiones.

- La construcción activa del proyecto profesional, que supone llevar a cabo lo planificado y, a partir de la experiencia, reevaluar y revisar esa propuesta.

- La flexibilidad, que exige la adaptación a circunstancias siempre dinámicas y, en ocasiones, inesperadas.

- La interacción constante con la realidad profesional que debe conocerse en profundidad (Ruiz Corbellá et al., 2014).

En definitiva, ser conscientes de que la inserción profesional es tanto un fenómeno dependiente de los contextos como de factores personales en los que cada uno puede intervenir: formación adquirida, estrategias de búsqueda de empleo, tiempo a invertir, etc. (ANECA, 2009; Suárez Lantarón, 2014; Torres-Valdés, 2018; Pineda i Herrero, 2018). Todo ello puede, y debe, ser abordado y desarrollado de forma prioritaria en esta materia de Prácticas Externas.

\section{UNA EXPERIENCIA DE INNOVACIÓN DOCENTE}

\section{Prácticas profesionales, una materia orientada al mercado laboral}

La materia de Prácticas Externas cobró un especial valor en el diseño del Grado en Educación Social, asignándola, de acuerdo a lo sugerido en el Libro Blanco del Título de Grado en Pedagogía y Educación Social (ANECA, 2005), un valor de 30 ECTS. Carga crediticia que también asumió esta titulación en la UNED, lo que respalda el convencimiento de la relevancia de esta asignatura en la formación de los educadores sociales. Su contenido se centró en el aprendizaje y desarrollo de las competencias que demanda el contexto profesional (Pineda i Herrero et al., 2018), al organizarse en espacios de formación dirigidos a la inmersión en un contexto laboral real en los que

(...) el estudiante realiza un trabajo de reflexión y comprensión respecto a la acción socioeducativa y profesional y los entornos donde se da. Al mismo tiempo, realiza un trabajo introspectivo, de autoconocimiento e identificación de sus capacidades y limitaciones poniendo en juego sus propias maneras de hacer (...) (Rosa, Ribera y Fernández, 2013, s.p).

En la UNED, esta materia de Prácticas Profesionales se organizó en 5 asignaturas secuenciadas a partir del segundo curso del Grado, con objetivos y contenidos específicos y con un valor de 6 ECTS cada una de ellas (Tabla 1). 
Tabla 1. Objetivos por asignaturas de la materia de Prácticas Externas del Grado en Educación Social. Facultad de Educación. UNED

\begin{tabular}{|c|c|c|c|c|}
\hline PP I & PP II & PP III & PP IV & PP V \\
\hline $\begin{array}{c}\text { Acercarse a la } \\
\text { realidad de los } \\
\text { principales con- } \\
\text { textos profesio- } \\
\text { nales a través de } \\
\text { la observación } \\
\text { sistemática de } \\
\text { los escenarios y } \\
\text { situaciones habi- } \\
\text { tuales de la prác- } \\
\text { tica profesional. } \\
\text { Conocer y } \\
\text { aplicar la me- } \\
\text { todología de la } \\
\text { observación y } \\
\text { la observación } \\
\text { participante. }\end{array}$ & $\begin{array}{c}\text { Adquirir los } \\
\text { instrumentos y } \\
\text { técnicas propios } \\
\text { de una práctica } \\
\text { profesional re- } \\
\text { flexiva. } \\
\text { Analizar una } \\
\text { institución, cen- } \\
\text { tro o experiencia } \\
\text { de buenas prác- } \\
\text { ticas Formar al } \\
\text { educador social } \\
\text { en contextos } \\
\text { profesionales } \\
\text { diferidos. } \\
\text { Conocer básica- } \\
\text { mente técnicas } \\
\text { y recursos que } \\
\text { contribuyen a } \\
\text { mejorar el de- } \\
\text { sarrollo técnico } \\
\text { de la profesión } \\
\text { (técnicas de } \\
\text { planificación, } \\
\text { intervención } \\
\text { educativa, de co- } \\
\text { municación, etc. } \\
\text { para el análisis } \\
\text { de la realidad). }\end{array}$ & \begin{tabular}{|c|} 
Realizar análisis \\
de la realidad \\
socioeducativa, \\
de acuerdo al \\
contexto de in- \\
tervención, con \\
espíritu crítico y \\
finalidad de mejo- \\
ra. Valorar crítica- \\
mente estrategias, \\
procedimientos y \\
herramientas pro- \\
pios del contexto \\
profesional en el \\
que se interviene. \\
Detectar y pro- \\
poner necesida- \\
des de actuación \\
preventiva e \\
intervención en \\
contextos espe- \\
cíficos de áreas \\
socioeducativas. \\
Diseñar y desa- \\
rrollar planes y \\
programas de \\
intervención, \\
elaborando y/o \\
utilizando los re- \\
cursos necesarios \\
para las mismas.
\end{tabular} & $\begin{array}{c}\text { Aplicar un di- } \\
\text { seño específico } \\
\text { en un contexto } \\
\text { socioeducativo. } \\
\text { Evaluar ese dise- } \\
\text { ño socioeduca- } \\
\text { tivo. }\end{array}$ & $\begin{array}{l}\text { Analizar crítica- } \\
\text { mente el propio } \\
\text { proceso de for- } \\
\text { mación. } \\
\text { Concretar los } \\
\text { puntos fuertes } \\
\text { y débiles pro- } \\
\text { pios del ámbito } \\
\text { profesional del } \\
\text { Educador Social, } \\
\text { analizando sus } \\
\text { implicaciones. } \\
\text { Analizar el con- } \\
\text { texto laboral / } \\
\text { Profesional del } \\
\text { Educador Social, } \\
\text { identificando sus } \\
\text { oportunidades. } \\
\text { Establecer un } \\
\text { proyecto profe- } \\
\text { sional propio. }\end{array}$ \\
\hline
\end{tabular}

Fuente: Elaboración propia a partir de los datos existentes en la web de la Facultad de Educación. UNED

En este diseño la asignatura Prácticas Profesionales $V$ se imparte en el último curso del Grado, asumiendo el objetivo de aprender a diseñar su Proyecto Profesional como educador/a social, clave para su empleabilidad e inserción profesional inmediata. Para ello, deberá realizar el diseño de ese Proyecto con criterios de coherencia, viabilidad y sostenibilidad. Se pretende facilitar la búsqueda de un punto de encuentro entre las expectativas de cada estudiante y las oportunidades externas reales, como elementos clave, no sólo para la integración sociolaboral, sino también para avanzar en su autorrealización personal (Sánchez-García, 2013; Guàrdia-Olmos et al., 2018). 
En esta asignatura entendemos el Proyecto Profesional como la "construcción personal e intencional basada en una planificación de futuro, sistemática, abierta, flexible y progresiva, dirigida al desarrollo de la carrera profesional y vital a lo largo de la vida" (Suárez-Ortega; Sánchez-García, Padilla-Carmona, 2014, p. 15). Para ello, nos apoyamos en el estudio y análisis de 3 ámbitos interrelacionados y secuenciados, contrastados continuamente (Tabla 2).

Tabla 2. Fases de desarrollo del Proyecto Profesional

\begin{tabular}{|c|c|c|}
\hline \multicolumn{2}{|r|}{ Bloque I } & Bloque II \\
\hline Actividad I & Actividad II & Proyecto Profesional \\
\hline Ámbito personal & Entorno socio-profesional & Diseño del Proyecto Pro- \\
\hline Competencias adquiridas & Profesiones emergentes & fesional \\
\hline Formación & Nichos de empleabilidad & \\
\hline Aspiraciones & Opción de empleo & \\
\hline Circunstancias personales & $\begin{array}{l}\text { Requisitos y condiciones del puesto } \\
\text { laboral }\end{array}$ & \\
\hline
\end{tabular}

Fuente: Elaboración propia

Esta secuenciación de un análisis crítico, interno y externo, facilita que la propuesta de cada Proyecto Profesional (Sánchez-García, 2013):

- Active la capacidad de definir o redefinir cada fase vital.

- Delimite la identidad profesional.

- Adquiera las destrezas y actitudes necesarias para la inserción laboral.

- Utilice los instrumentos y recursos requeridos en toda inserción laboral.

- De sentido a cada una de las decisiones.

- Planifique un itinerario con sentido.

- Sepa enfrentarse al cambio con una propuesta sólida y personal.

En definitiva, potenciar el papel activo de cada estudiante centrado en la autorreflexión, comprensión y análisis del entorno laboral y social confrontándolo con la realidad. Es decir, aportar contenidos, recursos y herramientas para ayudar a que cada estudiante se conozca a sí mismo, conozca las oportunidades del mundo laboral y formativo, y desarrolle las destrezas necesarias para tomar decisiones de cara a la organización de su vida profesional en un contexto específico (Rodríguez Moreno, 2003; Dapía Conde y Fernández González, 2016).

\section{La formación para la empleabilidad en la asignatura de Prácticas Profesionales $V$ del Grado en Educación Social. El diseño de la asignatura}

Tal como se acaba de mencionar, el diseño de la asignatura de Prácticas Profesionales $V$ se dirige a la elaboración de un Proyecto Profesional personal en el que el estudiante debe revisar y analizar el mercado de trabajo en el área de intervención 
socioeducativa elegida y en un contexto geográfico determinado, de acuerdo a sus intereses y formación. A la vez, debe identificar un puesto de trabajo, las funciones que deberá desempeñar y los requisitos de acceso que demandan. En suma, desarrollar y utilizar las destrezas, herramientas y actitudes necesarias para afrontar su inserción laboral desde un proyecto profesional y vital propio, de tal modo que sea capaz de conocerse a sí mismo, conocer las oportunidades del mundo laboral y formativo, y tomar decisiones de cara a la organización y desarrollo de su carrera profesional (Rodríguez Moreno, 2003). Lógicamente, este proyecto debe ser evaluado permanentemente para ser capaz de adecuarse a las situaciones cambiantes, tanto a nivel externo, el propio mercado de trabajo, como interno, los roles, circunstancias y formación de cada persona (Álvarez Pérez y López Aguilar, 2012).

El contenido de esta asignatura se organiza en 2 bloques estrechamente interrelacionados: el primero de ellos centra la actividad en el autoconocimiento profesional y conocimiento del área de intervención socio-profesional, y el segundo se dirige al diseño del proyecto profesional como proyecto vital.

Es importante comprender que los apartados del bloque I, Autoconocimiento y Conocimiento del área de intervención socio-profesional, se concretan, en cada estudiante, a través de la recogida de información de la trayectoria de formación personal, de experiencias previas, además del análisis de la situación laboral de los educadores sociales en un contexto determinado. Contenido que debe concretarse en datos y evidencias objetivas para la posterior planificación y toma de decisiones del proyecto profesional que se desarrolla en el bloque II de la asignatura. En este segundo apartado se perfila el documento más importante y complejo, ya que cada estudiante muestra en él su capacidad de organizar y analizar toda la información recogida en la primera fase. Esta información, así como su análisis, debe conducir a una toma de decisiones justificada y argumentada, que se traduce en el diseño de su proyecto profesional en función de sus posibilidades y la realidad del entorno. Esta decisión deberá acomodarse a una opción de trabajo: por cuenta propia, autoempleo, empleo público o promoción.

El objetivo es que el estudiante realice una aproximación a la realidad laboral, a las empresas que ofertan puestos de trabajo y que conozca los requisitos de acceso, las condiciones de los mismos y las funciones que deberá desempeñar. De esta forma, el estudiante se ve obligado a reflexionar sobre su proyecto profesional y a diseñar una estrategia coherente evaluando las exigencias de ese puesto laboral y su adecuación al mismo, aportando las evidencias que lo avalen, lo que deriva en una planificación específica de cara a alcanzar la opción elegida (Álvarez Pérez y López Aguilar, 2012; Dapía Conde y Fernández González, 2016; Pineda i Herrero et al., 2018; Guàrdia-Olmos et al., 2018).

\section{RESULTADOS}

\section{Resultados obtenidos del diseño de Prácticas Profesionales $V$}

Prácticas Profesionales V Ileva implantada desde el curso 2012-2013, por lo que disponemos de suficientes datos y trayectoria como para valorar los resultados de 
este diseño. Para ello, a partir de una metodología longitudinal "ex post-facto", analizamos los resultados apoyándonos en las calificaciones recibidas y los cuestionarios de satisfacción de los estudiantes. A la vez, los datos recogidos en los análisis de las actividades realizadas por los estudiantes y en las respuestas de estos al cuestionario de satisfacción de la asignatura, instrumento elaborado por la universidad, aportan datos y valoraciones que proyectan cómo se valora esta asignatura, junto con los puntos fuertes y las deficiencias que se detectan a la hora de plantear un proyecto profesional.

En la Tabla 3 se recogen los porcentajes de aprobados en cada convocatoria, y en la Figura 1 se puede valorar la evolución de la media obtenida.

Tabla 3. Matricula y $\%$ de aprobados en la convocatoria ordinaria y extraordinaria en los 5 cursos académicos

\begin{tabular}{cccc}
\hline $\begin{array}{c}\text { Curso } \\
\text { académico }\end{array}$ & $\mathbf{N}^{\mathbf{0}}$ matriculados & $\begin{array}{c}\text { \% Aprobados } \\
\text { Conv Ordinaria }\end{array}$ & $\begin{array}{c}\text { \% Aprobados Conv } \\
\text { Extraordinaria }\end{array}$ \\
\hline $2012-2013$ & 190 & $49 \%$ & $53,27 \%$ \\
\hline $2013-2014$ & 363 & $52,28 \%$ & $65,19 \%$ \\
\hline $2014-2015$ & 478 & $45,62 \%$ & $70,56 \%$ \\
\hline $2015-2016$ & 478 & $58,43 \%$ & $70,94 \%$ \\
\hline $2016-2017$ & 529 & $67,10 \%$ & $70 \%$ \\
\hline
\end{tabular}

Fuente: Elaboración propia

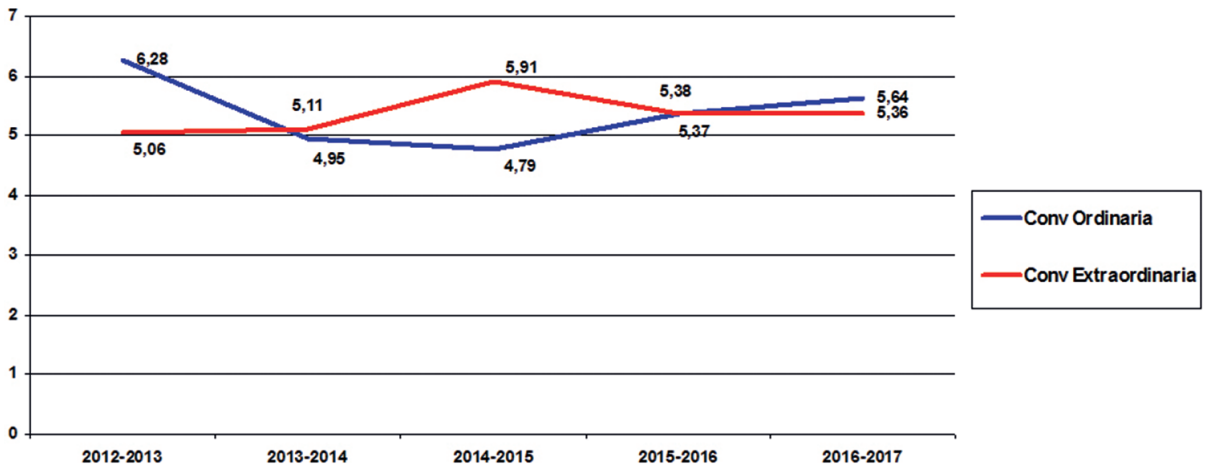

Figura 1. Media de los resultados globales por curso académico y convocatoria

En un primer análisis, estos resultados académicos muestran el bajo nivel de conocimientos y competencias que los estudiantes tienen en relación a la búsqueda de empleo en su propio sector, lo que nos indica un ámbito de formación al que las titulaciones no están prestando atención. Aunque debemos profundizar en este 
punto, es claramente una llamada de atención para abordar, de una u otra manera, la formación para la empleabilidad. Pero, ¿qué indican estos resultados?

\section{El perfil de nuestros estudiantes}

Los estudiantes que acceden a la UNED presentan un perfil diferenciado a los que se matriculan en otras universidades españolas, por lo que es necesario tenerlo en cuenta. Estamos ante un alumnado predominantemente femenino, en el que el $72,73 \%$ se sitúa entre los 36 y 50 años. Y que acceden al Grado para mejorar sus perspectivas profesionales bien como primer empleo, bien como mejora de su situación laboral. Para muchos de ellos se trata de su segunda carrera.

Al ser una asignatura que se imparte en cuarto curso, los estudiantes han superado más del $80 \%$ de los créditos cuando la cursan, por lo que se entiende que poseen ya conocimientos básicos como futuros educadores sociales. Además, como se recomienda, se matriculan en ella una vez superadas las asignaturas de Prácticas previas, por lo que el estudiante ya ha tenido, al menos, una primera experiencia en un entorno profesional real. Únicamente un pequeño porcentaje, un 10\%, tienen vinculación profesional con la Educación Social, ya que se matriculan en el Grado bien para poder continuar en el puesto laboral que desempeñan, bien para promocionar al estar contratados como titulados del Ciclo Superior de Formación Profesional de Servicios Socioculturales y a la Comunidad.

Por otro lado, un $45,45 \%$ de los estudiantes tienen experiencia laboral previa, aunque no relacionada con esta titulación. Y el 44,50\% restante, porcentaje significativo, no han tenido nunca contacto con el mercado laboral. Ahora, resulta preocupante que estos, tanto con trabajo como sin él, presentan un alto grado de desconocimiento sobre la situación real, posibilidades y vías de acceso o promoción en el mercado laboral en el que desean integrarse como educadores/as sociales. Una muestra de este desconocimiento es el alto porcentaje de estudiantes que decide el ámbito laboral en el que insertarse de acuerdo al área y colectivo en el que han realizado las prácticas externas presenciales, coincidiendo con otros estudios (Moreno Gené, 2012; Ruiz Corbellá et al., 2014). Muy pocos casos son los que analizan otras áreas u otras opciones. En este punto coincidimos con el informe de ANECA (2009) al afirmar que la inexperiencia laboral, el desconocimiento y el temor son factores que impiden una correcta búsqueda de empleo, lo que se comprueba en esta asignatura ante la falta de capacidad para abordar una estrategia de inserción laboral realista y efectiva.

La situación descrita avala la pertinencia del diseño de esta asignatura, ya que enfrenta a los estudiantes a cómo deben afrontar ese contacto con el mercado laboral en el que desean insertarse y a la búsqueda de un empleo acorde con sus intereses y posibilidades (Pineda i Herrero, 2018). Nos preocupa, además, este desconocimiento al traducirse en un acercamiento poco motivado y, como veremos, basado en cuestiones poco racionales, lo que coincide con lo que señala el informe de ANECA (2009), en el que destaca que los universitarios viven esta situación 
(...) con un cierto nivel de angustia, que en parte se debe al propio vértigo del cambio, pero sobre todo debida a la decepción con la formación recibida Esta decepción no tiene tanto que ver con una mala consideración de los contenidos estudiados o con la mala calidad de la formación recibida, sino ante todo, con la percibida falta de utilidad de los estudios para lograr una inserción laboral inmediata, con la inmensa brecha existente entre la universidad y el mercado laboral (ANECA, 2009, pp. 56-57).

De ahí que consideremos absolutamente necesario centrar una asignatura del Grado donde trabajar con ellos las competencias, los recursos y los contenidos necesarios para afrontar una inserción profesional eficaz en el área de la propia titulación.

\section{Sus motivaciones ante la búsqueda de empleo}

Para comprender la perspectiva de los estudiantes debemos conocer cuáles son sus motivaciones en esa búsqueda de empleo, cuál es la modalidad con la que pretenden esa inserción, el motivo por el que eligen un área de intervención u otra y el colectivo en el que quieren trabajar.

Como primer paso, en este proyecto profesional deben decidir cuál es la modalidad laboral que escogen para poder identificar las vías de acceso, requerimientos formales, necesidades formativas, etc. que deberán atender. La gran mayoría, más del $50 \%$, eligen el empleo por cuenta ajena al ser una de las vías que mejor conocen, especialmente gracias a las prácticas y/o al voluntariado. Como segunda opción se propone la Administración Pública, al ser considerada como un puesto estable y seguro, sin atender la dificultad real y la alta competitividad de estas convocatorias, no planteándose inicialmente otras alternativas.

La elección de emprendimiento se encuentra en el tercer puesto por orden de preferencia. Se trata sin duda la más compleja de todas las opciones, ya que requiere un planteamiento vital radicalmente distinto, en el que se diferencian las personas emprendedoras entre aquellas que inician un negocio al identificar una oportunidad no explotada en el mercado, y aquellas que lo emprenden ante la falta de alternativas laborales (GREM, 2018). Sea una u otra, “(...) es vital seguir apostando por el mejoramiento de las condiciones del ecosistema emprendedor e innovador español a través de la colaboración, el compromiso y la co-creación entre los diversos actores sociales, económicos y políticos vinculados al mismo en el mercado" (GREM, 2018, 121). El emprendimiento debería ser una alternativa muy meditada, aunque no siempre sea así. Además, es reseñable que el 18\% de las personas que deciden emprender lo hacen para cambiar su vida profesional y un $1 \%$ aduce que estaba desempleado. Esta situación está reflejada en los estudiantes que escogen esta opción, junto con la necesidad de formarles en este modo de inserción. Por último, en cuarto lugar, aparece la vía de promoción en la institución en la que trabajan.

En cuanto a los motivos que estos estudiantes manifiestan para fundamentar su decisión se alejan mucho de lo que se espera de los alumnos de último curso: un proceso reflexivo que les ayude a tomar decisiones de manera argumentada. Como se desprende en la Figura 2, la mayoría se limita a justificar su elección en unos intereses personales, sin contrastarlos con su viabilidad y sostenibilidad en el contexto en el 
que viven y con las salidas profesionales que les facilita el Grado, cuestión que es similar a la indicada por otros autores (Pineda i Herrero et al., 2018; Guàrdia-Olmos et al., 2018).

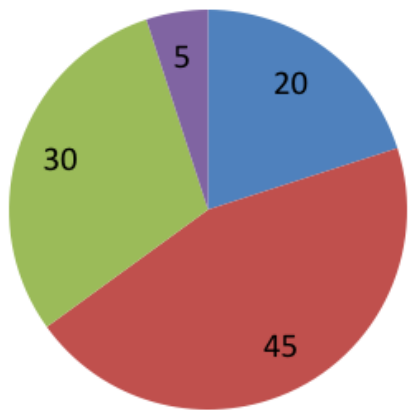

Reflexión
argumentada
Vocación y
preferencias
Lugar de realización
de las prácticas
Trabajo actual

Figura 2. Motivos para la toma de decisiones en torno al proyecto profesional

Lo mismo sucede en cuanto al colectivo con el que desean trabajar, en el que predomina el de "menores y adolescentes en riesgo de exclusión social", seguido del de "personas con discapacidad", al ser los 2 campos en los que se centran prioritariamente las instituciones con las que la UNED tiene establecidos convenios para la realización de las Prácticas en este Grado. Este criterio coincide con lo indicado en el Informe McKinsey \& Co. (2013), en el que destacan que en nuestros universitarios la realidad que conocen parece primar a las demandas profesionales emergentes y a la movilidad. No se plantean realizar una búsqueda de otras opciones en la localidad en la que viven, o en el entorno cercano, o la posibilidad de movilidad a otras regiones y/o países donde podrían encontrar mejores posibilidades de empleo. O la capacidad de identificar nuevas demandas o necesidades sociales, punto en el que especialmente la capacidad emprendedora resulta ser una de las opciones más débiles de nuestros estudiantes, y que aún no hemos sabido incorporarla a nuestras aulas (Peña et al., 2018).

\section{Principales barreras para diseñar una estrategia efectiva de empleo}

A la hora de buscar trabajo por cuenta ajena, los estudiantes mantienen estrategias poco activas. La opción "echar el curriculum y esperar" sigue siendo la estrategia más común entre aquellos que buscan empleo por cuenta ajena, junto con la de mantenerse informados a través de portales genéricos de empleo. A esto se une el desconocimiento que manifiestan para diseñar su Proyecto Profesional y que continúen resaltando el temor ante la situación de crisis económica para avalar sus decisiones (Pineda i Herrero et al., 2018). Este motivo es el que aducen mayoritariamente los estudiantes que optan por el empleo en la Administración Pública, sin tener en cuenta 
que el momento de recortes en este tipo de convocatorias ha ocasionado que muchas de estas no sean convocadas con una declarada periodicidad. Ante esta situación de incertidumbre los estudiantes siguen optando por esta vía como estrategia segura, sin preguntarse por el índice de dificultad y de competitividad en este tipo de pruebas. A la vez desconocen, o no tienen en cuenta, la diferencia entre un concurso, concursooposición, concurso de méritos, bolsa de trabajo... lo que conlleva graves errores e incoherencias a la hora de diseñar este proyecto, además de percibirse una gran ingenuidad ante el análisis de contenido que llevan a cabo en esta opción.

Los estudiantes que plantean la opción del emprendimiento podríamos decir que se agrupan en dos grupos, con excepciones. El primero de ellos está compuesto por estudiantes con experiencia previa que plantean un proyecto coherente y con una adecuada justificación de la viabilidad. Y un segundo grupo compuesto por personas que tienen una idea de negocio en ocasiones muy idealizada, que desconocen la complejidad que implica el establecimiento de una empresa propia. En este segundo grupo se detectan lagunas formativas que deberían contemplarse en una formación universitaria: entre otros contenidos, la formación sobre las posibilidades de creación de una empresa, emprendimiento social, normativa legal, alianzas estratégicas, etc.

En cambio, los estudiantes que optan por la promoción en la propia empresa o institución son los que obtienen mejores resultados. Resulta lógico dado que conocen bien su entorno profesional, están motivados para alcanzar un puesto de mayor responsabilidad y saben cómo lograrlo.

En cuanto a carencias más significativas en este campo destacan las competencias digitales específicas para el desarrollo profesional (Torres-Valdés et al., 2018; Guàrdia-Olmos et al., 2018). Desconocen el uso de las redes sociales para el networking, las redes más relevantes en su ámbito profesional, tanto para encontrar empleo, promocionar profesionalmente o promover una idea de negocio. No saben elaborar un Curriculum Vitae, una carta de presentación o preparar una entrevista de trabajo. $\mathrm{O}$ como mostrar el dominio de las soft skills que demandan los empleadores. Adolecen de la proactividad necesaria para hacer llegar propuestas profesionales a empresas y/o instituciones que podrían valorarlas.

\section{El nivel de satisfacción en esta asignatura}

Más allá de las dificultades y del alto porcentaje de suspensos, los estudiantes valoran muy positivamente la asignatura, como comprobamos a partir de los resultados del cuestionario de satisfacción. Bien es cierto que les cuesta mucho comprenderla, al reclamar de ellos actividades que se escapan del tradicional diseño de las asignaturas universitarias: trabajar con los recursos diversos en cada una de las fases: búsqueda de información, entrevistas con agentes clave, recogida de los datos necesarios, análisis de contenido, etc. Además en esta asignatura parte del conocimiento es generado por los propios estudiantes al analizar el mercado laboral en el que desean insertarse, encontrando y justificando nuevos nichos de empleabilidad, diseñando prototipos de empresas con valor social, cuestión que conllevan una tarea en la que deben movilizar gran parte de las competencias adquiridas a lo largo de sus estudios, 
lo que supone un enorme grado de exigencia. De ahí que, a lo largo de estos cursos académicos, se haya enriquecido el material didáctico con videoclases, enlaces a cursos disponibles en la web, entrevistas grabadas a expertos, documentos, oferta de cursos en línea, etc., junto con la atención tutorial personalizada propia de la UNED.

En la Tabla 4 se recoge cómo la mayoría de nuestros estudiantes valoran su paso por la asignatura con un nivel medio-alto, al situarse todos los porcentajes por encima de $60 \%$. Muchos de ellos manifiestan que es la primera vez que se enfrentan a un análisis dirigido a su inserción laboral. Desconocen los medios y herramientas para llevarlo a cabo, las ayudas y recursos con los que pueden contar, tanto de la UNED como universidad como de otras instituciones. No obstante, a pesar de las dificultades, les supone, según ellos mismos, un aprendizaje muy útil y satisfactorio de cara a su futuro. En definitiva, expresan que ya saben qué tienen que hacer, cómo llevarlo a cabo y por qué en su proceso de inserción laboral una vez finalizado el Grado.

Tabla 4. Valoración de los objetivos de la asignatura por parte de los estudiantes

\begin{tabular}{|c|c|c|c|c|c|}
\hline Objetivos de la asignatura & Muy Bajo & Bajo & Medio & Medio Alto & Muy Alto \\
\hline $\begin{array}{l}\text { Analizar críticamente el pro- } \\
\text { pio proceso de formación }\end{array}$ & $4.76 \%$ & $0.00 \%$ & $9.52 \%$ & $80.95 \%$ & $4.76 \%$ \\
\hline $\begin{array}{l}\text { Estudiar el contexto laboral- } \\
\text { profesional del Educador } \\
\text { Social identificando sus opor- } \\
\text { tunidades }\end{array}$ & $4.76 \%$ & $0.00 \%$ & $28.57 \%$ & $61.90 \%$ & $4.76 \%$ \\
\hline $\begin{array}{l}\text { Identificar los puntos fuertes } \\
\text { y débiles propios relativos al } \\
\text { ámbito profesional elegido }\end{array}$ & $4.76 \%$ & $0.00 \%$ & $28.57 \%$ & $61.90 \%$ & $4.76 \%$ \\
\hline $\begin{array}{l}\text { Diseñar un proyecto profesio- } \\
\text { nal propio }\end{array}$ & $4.76 \%$ & $0.00 \%$ & $19.05 \%$ & $71.43 \%$ & $4.76 \%$ \\
\hline
\end{tabular}

\section{Conclusiones}

Aunque aún estamos analizando esta experiencia desde los resultados obtenidos a lo largo de estos 5 cursos, si avanzamos la necesidad y el valor de los conocimientos y destrezas de empleabilidad en la trayectoria formativa de nuestros estudiantes de Grado. La asignatura de Prácticas Profesionales $V$ les facilita el análisis del mercado laboral en una determinada área de intervención, lo que les aporta datos sobre la situación real de su campo profesional para poder optar a él con mayor garantía de éxito. Muchas frustraciones en la búsqueda de empleo se derivan del desconocimiento del funcionamiento del mercado de trabajo, y, en concreto, el de su campo profesional, por lo que la búsqueda de información en diferentes fuentes les ha posibilitado conocer los canales de información, además de poder analizar los datos reales de un campo de intervención, de tal forma que avale de forma realista sus decisiones.

Si la empleabilidad es uno de los objetivos de la formación universitaria, resulta imprescindible mejorar las posibilidades de inserción laboral de nuestros titulados, teniendo en cuenta la situación del mercado laboral y también las necesidades de 
una sociedad en constante cambio. Por ello, se hace necesario el análisis de las propias posibilidades pero también del contexto socio laboral con una mirada crítica, como futuros egresados y como ciudadanos comprometidos con la mejora personal pero también de la comunidad.

El emprendimiento, especialmente con carácter social, sigue siendo una faceta poco desarrollada y que debe favorecerse, por lo que atenderlo desde cada titulación, junto con las acciones llevadas a cabo en otras instancias de la universidad, resultarán claves para nuestros egresados. Sin duda, entendemos que esta asignatura colabora definitivamente en la formación para una inserción laboral efectiva.

En suma, aunque inicialmente no son conscientes, nuestros estudiantes se enfrentan a una asignatura compleja en la que deben poner en juego un gran número de competencias tanto genéricas como específicas del Grado, junto con las propias para la empleabilidad. Destaca la gran dificultad que manifiestan para analizar con rigor y espíritu crítico la propia situación y la del contexto laboral elegido, lo que condiciona esa toma de decisiones. Esto deriva en que no sepan elaborar una propuesta coherente y con una estrategia clara y definida que les permita alcanzar su objetivo: obtener el puesto de trabajo seleccionado. Además, manifiestan dificultades para expresar sus ideas, argumentar decisiones acordes a la documentación e información que han recogido y analizado a lo largo de la asignatura. Este resultado pone de manifiesto que en la formación de nuestros titulados no puede dejarse de lado competencias orientadas a facilitarles una positiva inserción en el mercado profesional. El complejo contexto sociolaboral exige de las universidades una formación que permita a los estudiantes la toma de decisiones conscientes, fundamentadas para mejorar sus opciones profesionales y evitar trayectorias fallidas, con el enorme coste social y económico que ello conlleva. Favorecer nuevas vías de relación profesional (más allá de los tradicionales), nuevas maneras de relacionarse con las empresas existentes o de imaginar otras opciones viables de actividad profesional. Todo ello exige la movilización consciente de competencias, algunas presentes en los títulos y otras que hay que considerar, para avanzar y hacer avanzar la vertiente profesional activa de nuestros estudiantes, futuros egresados.

\section{REFERENCIAS BIBLIOGRÁFICAS}

Álvarez Pérez, P. R. y López Aguilar, D. (2012). Centralidad del trabajo y estabilidad del proyecto profesional y vital. Revista Española de Orientación y Psicopedagogía, 23(1), 13-25.

ANECA (2005). Libro Blanco: Título de Grado en Pedagogía y Educación Social. Madrid: ANECA.

ANECA (2009). Los procesos de inserción laboral de los titulados universitarios en España. Madrid: ANECA.

Beneitone, P., González, J. y Wagenaar, R. (2014). Meta-perfiles y perfiles. Una nueva aproximación para las titulaciones en América Latina. Bilbao: Universidad de Deusto. 
Caballero Fernández, G., López-Miguens, M. J. y Lampón, J. F. (2014). La universidad y su implicación con la empleabilidad de sus graduados. Revista Española de Investigaciones Sociológicas, 146, 23-46.

Dapía Conde, M. D. y Fernández González, M. R. (2016). La Búsqueda Activa de Empleo en el colectivo universitario: conocimientos y prácticas. Revista Española de Pedagogía, 265, 517-542.

Enríquez, A. y Rentería, E. (2007). Estrategias de aprendizaje para la empleabilidad en el mercado del trabajo de profesionales recién egresados. Universitas Psychologica, 6(1), 89-103.

Fundación CyD (2012). Informe CyD 2011. Barcelona: Fundación Conocimiento y Desarrollo. Recuperado de https://www.fundacioncyd.org/publicaciones-cyd/ informe-cyd-2011/.

González Lorente, C. y Martínez Clares, P. (2016). Expectativas de futuro laboral del universitario de hoy: un estudio internacional. RIE. Revista Investigación Educativa, 34(1), 167-183.

Guàrdia-Olmos, J., Peró-Cebollero, M., Martínez-Ricart, M., Cañete-Masse, C., Turbany-Oset, J. y Berlanga-Silvente, V. (2018). Employability of recent graduates. Opinions of Catalonian companies and institutions. Educación XX1, 21(2), 131154.

Hernández Armenteros. J. y Pérez García, J. A. (dir) (2017). La Universidad Española en cifras. Madrid: CRUE. Recuperado de http://www.crue.org/Documentos\%20compartidos/Publicaciones/Universidad\%20Española\%20en\%20cifras/ UEC_Digital_WEB.pdf.

McQuaid, R. W. y Lindsay, C. (2005). The Concept of Employability. Urban Studies, 42(2), 197-219.

McKinsey \& Co. (2013). Empleo juvenil en España. Recuperado de: https://goo.gl/ AvPCzf/.

Michavila, F., Martínez, J. M., Martín-González, M., García-Peñalvo, F. J. y CruzBenito, J. (2016). Barómetro de Empleabilidad y Empleo de los Universitarios en España, 2015 (Primer informe de resultados). Madrid: Observatorio de Empleabilidad y Empleo Universitarios.

Ministerio de Educación y Cultura (MECU) (2014). Marco de Cualificaciones. Disponible en: https://goo.gl/FkezRp.

Moreno Gené, J. (2012). La nueva regulación de las prácticas académicas externas de los estudiantes universitarios: la potenciación de la finalidad formativa y de mejora de la empleabilidad. Estudios financieros. Revista de trabajo y seguridad social, 349, 5-62.

Peña, I., Guerrero, M., González-Pernía, J. L. y Montero, J. (2018). GEM, Global Entrepreneurship Monitor. Informe España. 2017-2018. Santander: Universidad de Cantabria. 
Pineda-Herrero, P., Agud-Morell, I. y Ciraso-Calí, A. (2016). Factores que intervienen en la inserción laboral de los titulados en educación en tiempos de crisis: un estudio sobre Cataluña. Revista de Educación, 372, 141-168. http://doi. org/10.4438/1988-592X-RE-2015-372-318.

Pineda i Herrero, P., Ciraso Calí, A. y Armijos Yambay, M. (2018). Competencias para la empleabilidad de los titulados en Pedagogía, Psicología y Psicopedagogía: un estudio comparativo entre empleadores y titulados. Revista española de pedagogía, 270, 313-333.

Rodríguez Moreno, M. L. (2003). Cómo orientar hacia la construcción del proyecto profesional. Bilbao: Desclée de Brouwer.

Rosa, G., Ribera, G. y Fernández, C. (2013). Las competencias transversales y la empleabilidad. Planteamiento curricular de los Grados de Educación Social y de Trabajo Social [mensaje en un blog]. Recuperado de https://www.educaweb.com/ noticia/2013/11/18/competencias-transversales-empleabilidad-planteamientocurricular-grados-educacion-social-trabajo-social-7899/.

Ruiz Corbellá, M. y Quintanal, J. (2012). Cómo organizar las prácticas profesionales y la inserción profesional de los egresados. En A. de la Herrán y J. Paredes, Promover el cambio pedagógico en la universidad (pp. 169-190). Madrid: Pirámide.

Ruiz-Corbella, M., García-Blanco, M., Bautista-Cerro, M. J. (2014). ¿La universidad debe de formar para la empleabilidad? Una respuesta desde el Grado en Educación Social. Procedia. Social and Behavioral Sciences, 139, 26-34. http://doi. org/10.1016/j.sbspro.2014.08.016.

Sánchez-García, M. F. (Coord.) (2013). Orientación Profesional y Personal. Madrid: UNED.

Suárez Lantarón, B. (2013). Servicios de Orientación profesional universitarios: estudio descriptivo. Red U. Revista de Docencia Universitaria, 11(2):27-42.

Suárez Lantarón, B. (2014). La universidad española ante la empleabilidad de sus graduados: estrategias para su mejora. Revista Española de Orientación y Psicopedagogía, 25(2), 90-110.

Suárez-Ortega, M., Sánchez-García, M. F. y Padilla-Carmona, M. T. (2014). Aprender a diseñar la carrera profesional y a gestionar el talento emprendedor como vías para evitar la exclusión socio-laboral. Revista Iberoamericana de Producción Académica y Gestión Educativa. 1(2). Recuperado de http://www.pag.org.mx/index. php/PAG/article/view/139/187.

Torres-Valdés, R. M., Santa Soriano, A. y Lorenzo Álvarez, C. (2018). Resignification of educational e-innovation to enhance opportunities for graduate employability in the context of new university degrees. NAER: Journal of New Approaches in Educational Research, 7(1), 70-78.

UNED (2009). Grado en Educacion Social. Guía de la Materia Prácticas Profesionales. Recuperado de https://goo.gl/7iKAaL. 